\title{
Penile cancer: potential target for immunotherapy?
}

\author{
Joren Vanthoor ${ }^{1} \cdot$ Gigi Vos $^{1} \cdot$ Maarten Albersen ${ }^{1}[$ \\ Received: 16 July 2020 / Accepted: 23 October 2020 \\ (c) Springer-Verlag GmbH Germany, part of Springer Nature 2020
}

\begin{abstract}
Purpose Penile cancer $(\mathrm{PeCa})$ is a rare malignancy with a poor prognosis in advanced disease. There is still a limited understanding of the biological mediators that are important in the prognosis and therapy of the disease. This review aims to provide a summary of the immune micro-environment, molecular oncogenesis and the role of HPV in the disease applying to the potential of the use of immunotherapy.

Methods Narrative, non-systematic review based on publications retrieved by PubMed and EMBASE search.

Results The molecular mechanisms underlying penile carcinogenesis are complex, and human papillomavirus (HPV) infection is a well-characterized driver of penile cancer. Up to $50 \%$ of the penile carcinomas are HPV related. There is potential to improve prevention, treatment and follow-up strategies pertaining to the role of HPV in penile cancer. Immune response modifiers such as toll-like receptor agonists are being used in a topical fashion for penile intraepithelial neoplasia while immune checkpoint inhibitors are currently under clinical investigation for its application in penile cancer.

Conclusions The knowledge of prognosis-relevant biological pathways in penile cancer is expanding. HPV plays an important role in the carcinogenesis. This can lead to the identification of therapeutic targets which could significantly influence the prognosis of advanced penile cancer. Clinical trials are being conducted to pave the way for immune-modifying treatment modalities.
\end{abstract}

Keywords Penile cancer $\cdot$ Human papillomavirus $\cdot$ Imiquimod $\cdot$ Immunotherapy

\section{Introduction}

Primary penile cancer is a rare disease with an incidence of about 1/100,000-1,000,000 men annually [1]. There are approximately 26,000 new cases diagnosed each year globally, and it accounts for $0.4-0.6 \%$ of all malignancies in the Western world $[2,3]$. The incidence varies strongly among different geographic areas, with an incidence up to $6 \%$ of malignancies in developing countries [4]. More than $95 \%$ of the penile cancer tumors are squamous cell carcinoma (SCC), causing significant morbidity and mortality. Important risk factors include HPV infection, lack of circumcision, phimosis, obesity, lichen sclerosus, inflammation, smoking, previous UVA phototherapy and socioeconomic status [2, 5].

Joren Vanthoor and Gigi Vos are co-first authors.

Maarten Albersen

Maarten.albersen@uzleuven.be

1 Department of Urology, University Hospitals Leuven, Herestraat 49, 3000 Leuven, Belgium
The rarity of this disease creates a challenge for treating physicians. There is still only a limited understanding of the biological mechanisms, micro-environment and biomarkers in penile cancer. As HPV DNA is detected in half of the squamous cell carcinoma of the penis, this infection is strongly associated with $\mathrm{PeCa}$ specimens [6]. This review aims to provide a summary of the molecular oncogenesis and the role of HPV in the disease. Further, we discuss the potential for therapeutic options modulating viral infection and host immune responses in premalignant to advanced penile cancer $[7,8]$.

\section{Molecular oncogenesis of penile cancer}

The molecular mechanisms underlying penile carcinogenesis are complex and still largely unknown. As recognized by the WHO, there are two molecular pathways of etiology: the HPV-dependent and the HPV-independent carcinogenesis [9], as shown in Fig. 1. A final common pathway has been suggested. 


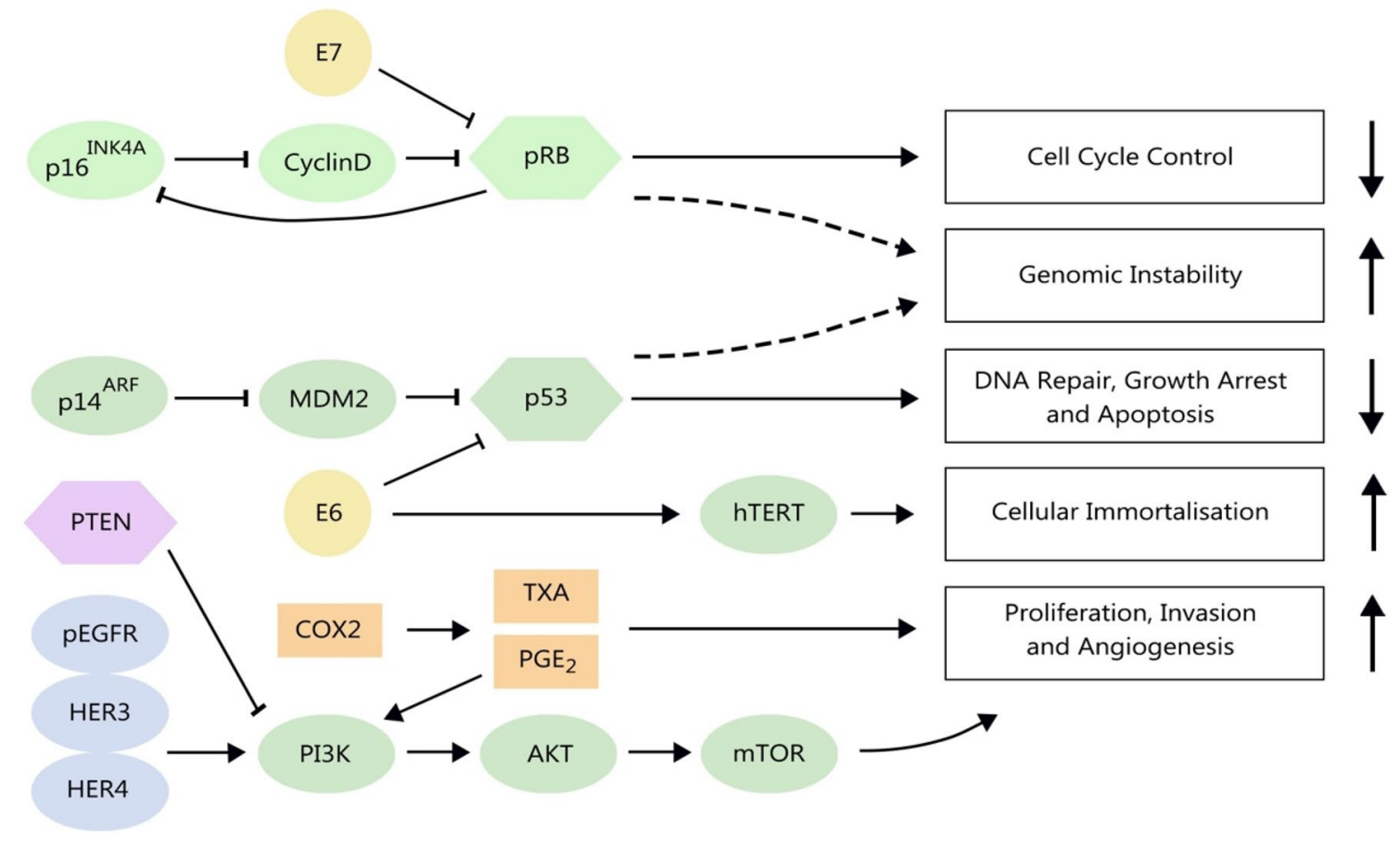

Fig. 1 Schematic diagram of the oncogenesis of penile cancer

\section{HPV-dependent penile carcinogenesis}

A recent meta-analysis of 52 studies $(n=4199)$ found that 50.8\% (95\% CI 44.8-56.7) of the penile carcinomas are HPV related [10]. High-risk HPV particles infect the basal cells of the epithelial mucosa, via micro-abrasions and specific receptors. The integration of HPV DNA into the host cell genome leads to the overexpression of viral oncoproteins (such as E6 and E7) [11].

Oncoprotein E7 interacts with the retinoblastoma tumor suppressor protein (pRB), resulting in the release of transcription factor E2F. This results in the activation of DNA synthesis genes and the cell cycle becomes uncontrolled. Due to the disturbance of the negative feedback via $\mathrm{pRB}$, an overexpression of P16 INK4A can be observed [11]. Hence, p16 INK4A may be used as a surrogate marker for the detection of active high-risk HPV infection in penile cancer [11, 12]. Oncoprotein E6 inactivates the tumor suppressor protein p53, leading to a disruption of DNA repair, growth arrest and apoptosis. It also supports cellular immortalisation by activating telomerase via c-myc-induced human telomerase reverse transcriptase (hTERT) expression [13].

In addition, both oncoproteins can interfere with the process of mitosis, inducing genomic instability [11]. Low-risk HPV are often found in penile cancer; however, they do not cause a significant dysregulation of tumor suppressor protein expression [11].

\section{HPV-independent penile carcinogenesis}

The HPV-independent penile carcinogenesis and its underlaying genetic mechanisms are less well understood. Chronic inflammation is a recognized mediator, in which cyclooxygenase-2 has an important role. This enzyme is overexpressed in premalignant and malignant penile lesions [14]. The subsequent overproduction of thromboxanes and prostaglandins (mostly $\mathrm{PGE}_{2}$ ) leads to proliferation, invasion and angiogenesis via activation of multiple pathways [11].

Advances in genetic sequencing revealed multiple somatic genetic alterations as a mechanism of carcinogenesis in penile cancer. The dysregulation of major tumor suppressor pathways (p16INK4A/cyclin D/RB and p14ARF/ MDM2/p53 pathways) also occurs via non-HPV-related mechanisms. The p16INK4A gene can be inactivated by loss of heterozygosity or silencing via DNA methylation [11]. Downregulation of p16INK4A due to the overexpression of BMI-1 polycomb gene has also been described in penile cancer $[11,15]$. There is a significant association between the loss of p16INK4A immunoexpression and lymph node metastasis and disease recurrence [16, 17]. TP53 mutation can result in a lack of p53 or excess negative regulation of this tumor suppressor protein in HPV-independent penile cancer [11]. Mutation of the p53 gene can result in the absence of its protein or lead to increased expression of an inactive p53 mutant protein [11, 18]. 
Another studied course in the carcinogenesis of penile cancer is the PI3K-AKT-mTOR pathway. Various cohort studies found an overexpression of mTOR and its stimulators (pEGFR, HER3 and HER4) in penile cancer tissue $[19,20]$. Interestingly, an association with HPV-negative status was observed. However, other study groups could not show this increase in immunoexpression compared to normal penile tissue [21]. Per contra, reduced or absent expression of PTEN, a tumor suppressor gene and inhibitor of the PI3K-AKT-mTOR pathway, has been reported $[19,21]$. For the latter no link with the tumor HPV status could be detected. To detect the true contribution of the PI3K-AKT-mTOR pathway and its potential therapeutic significance, further research is necessary.

\section{Human papillomavirus in penile cancer}

HPV infection is one of the most important risk factors in the development of penile cancer. The risk of infection increases with a previous history of genital warts and with the number of sexual partners. In a recent publication, Hansen et al. indicated an increase in the number of diagnoses amongst younger men ( $\leq 64$ years of age), which may be linked with augmented exposure to HPV in this population group [22]. The oncogenic strains HPV 16, 18, 31 and 33 have been strongly associated with penile cancer. As mentioned previously, the different pathways in the pathogenesis has led to a WHO classification of HPV-dependent and -independent penile cancer [9]. Verrucous carcinoma, pseudoglandular carcinoma, pseudohyperplastic carcinoma, adenosquamous carcinoma and papillary squamous cell carcinomas are classified as non-HPV-related. Basaloid squamous cell carcinoma, papillary-basaloid carcinoma, warty(-basaloid) carcinoma, clear-cell carcinoma and lymphoepithelioma-like carcinoma are HPV-related tumors. Prevention and treatment of HPV infection could be a promising target to reduce morbidity and mortality of the disease.

Precursor penile lesions are also classified into undifferentiated (HPV related) and differentiated (non-HPV related) penile intraepithelial neoplasia (PeIn) [23]. HPVrelated lesions account for approximately $25 \%$ of the precursor lesions [11]. They are usually erythematous and flat, found on the penile glans and/or foreskin with basaloid/ warty morphological features. The lesions typically display P16 INK4A positivity, and can progress to basaloid/warty or usual type penile cancer. It has been estimated that, if left untreated, the risk of malignant transformation is $30 \%$ [24].

\section{Prognostic impact}

The prognostic significance of HPV status in penile cancer is still uncertain. A contemporary review of 2017 evaluated eight studies addressing the issue [25]. The studies with the largest cohorts (212 and 171 patients) showed a favorable survival for HPV-positive patients compared to HPV-negative groups. The role of HPV status was inconclusive or negative in other trials [26].

In another meta-analysis of 2018, including 29 studies and 649 men with penile cancer, the prognostic significance of HPV and p16 status on survival was assessed [27]. They concluded a significantly better disease-specific survival for HPV or p16-positive penile cancer. However, HPV and p16 status had a significant prognostic impact on overall survival. The authors indicated that HPV DNA and p16 expression are important predictive markers for survival. They proposed that the presence of viral infections could confer an increased immune reaction and a more favorable molecular profile, which results in HPV-positive cancers being less aggressive.

In vulvar and oropharyngeal squamous cell carcinoma, other HPV-associated tumors, HPV is established as a prognostic factor [28, 29]. Hölters et al. could not extend this to penile cancer in a cohort of 121 patients; HPV itself was not identified as a prognostic factor [30]. However, the prognostic role of HPV infection in the different subtypes of the disease remains unclear. Histological subtype and lymph node status are by far the most predictive factors.

\section{Therapeutic impact}

Lifestyle modifications can be made to minimalize the risk of genital HPV transmission. The risk of infection increases with multiple sexual partners and early age of first intercourse, which augments the risk for penile cancer three- to fivefold [31]. Based on the data from the HIM (HPV in men) study, tobacco and smoking appear to be independent risk factors [32, 33]. Circumcision in childhood reduces the risk of invasive penile cancer. The effect of circumcision could be largely mediated by elimination of phimosis, rather than a decreased risk of HPV transmission.

Prophylactic HPV vaccination is another primary prevention strategy. The vaccines are L1 virus-like particle (VLP) vaccines; they resemble copies of the capsid protein of HPV [31]. Following HPV vaccines are currently available: bivalent (HPV 16, 18), quadrivalent (HPV 6, 11, 16, 18) and nonavalent (HPV 6, 11, 16, 18, 31, 33, 45, 52, 58). The vaccines do not contain HPV DNA, so they are not infectious nor oncogenic. As a result, no serum antibodies are developed and established cellular HPV infections are not targeted. HPV vaccination has led to a significant decrease in HPV-associated infections and cancers. In men, similar data have yet to be reported.

In a randomized, placebo-controlled, double-blind trial investigating 4065 healthy boys and men aged 16 to 26 years old, the quadrivalent vaccine demonstrated an efficacy of 
65.5\% (95\% CI 45.8-78.6) for HPV 6, 11, 16 of 18 related lesions [34]. Nevertheless, no significant reduction in external genital lesions associated with HPV-16 or HPV-18 were observed. More and more health institutions are convinced of the benefit of HPV vaccination in children (9-14 years) of both sexes. Educations programs are needed to raise awareness for the role of HPV in PeCa [35]. Additionally, vaccination programs for pre-pubertal boys and young men are expected to reduce HPV-associated cancers in female as well. Large population-based studies are necessary to evaluate the true impact of HPV vaccination.

As shown before, HPV E6 and E7 oncoproteins are crucial in the malignant transformation of HPV-associated penile cancer. These proteins are of special interest for the development of targeted therapies [36]. Promising results have been reported for E6/E7 targeted therapy in HPV16/18 cervical cancer [37]. Currently, there is no targeted treatment for HPV-related PeCa. A phase 1 trial including penile carcinoma explores the role of HPV-specific T cells administered with nivolumab [38]. Another trial is investigating the effect of vaccines with synthetic plasmids targeting HPV-12 and HPV-18 E6 and E7 proteins.

\section{Micro-environment and biomarkers}

For efficacious immunotherapy treatment, a better understanding of immunomodulatory factors, and thus the tumor micro-environment (TME) is critical.

\section{Tumor mutational burden}

The tumor mutational burden (TMB) is a marker for the sensitivity to immune checkpoint inhibitors. A high TMB is associated with a more effective immunotherapy response.
In penile cancer TMB values between 3.6 and 4.5 mutations/Mb have been reported. A TMB of $>20$ mutations/ $\mathrm{Mb}$, a surrogate for microsatellite instability, was reported in $6 \%$ of the specimens in penile cancer [39]. These data argue against a high mutational load in penile cancer, and are comparable to data in cervical carcinoma. This is in contrast to head and neck carcinoma, and especially much lower than for melanoma and bladder cancer [39, 40]. In a recent comparative study, Jacob et al. demonstrated a significantly higher TMB in predominantly ultraviolet light-exposed metastatic cutaneous SCC than in metastatic penile SCC [41]. The authors concluded that metastatic penile SCC is a unique subtype of SCC with distinctive genomic features.

A retrospective cohort study $(n=75)$ found an intact mismatch repair (MMR) system and microsatellite stability in penile cancer, as one would expect with a low TMB [42]. Interestingly, when analyzing the somatic mutational landscape of penile cancer via whole exome sequencing, high viral load tumors ( $>1 \mathrm{HPV}$ copy/cell via qPCR) had a significantly lower mutational load compared to HPV-negative tumors [43]. HPV-related cellular alterations are assumed to increase the tumor foreignness, which might act as targets for the first promising results of an immune checkpoint blockade therapy in penile SCC. This is encouraging for future clinical and basic research efforts [42].

\section{Tumor micro-environment}

Tumor-infiltrating lymphocytes (TILs) are an important component of the tumor micro-environment (Fig. 2). In other malignancies, it has been shown that T-cell infiltration has an important role in anti-tumor immunity and is associated with better survival [42].

Likewise, increased CD8 + T-cell and FoxP3 regulatory T-cell counts have been found in the stroma of penile cancer
Fig. 2 Tumor micro-environment overview

\section{Tumor hostile components}

\section{Tumor promoting components}

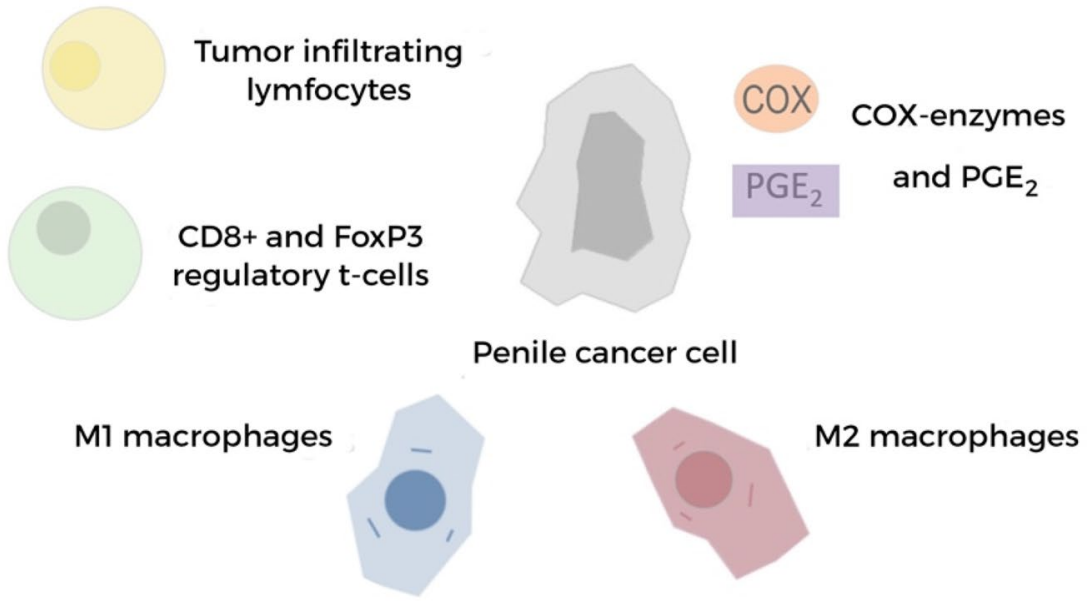


tissue $[42,44]$. Higher numbers were described in HPVpositive versus HPV-negative penile cancer, indicating a stronger cytotoxic immune response and immune escape in HPV-related penile cancer [45, 46].

Tumor-associated macrophages (TAMs) include both the M1 macrophages, which are involved in tumor suppression, and the M2 macrophages, which have a tumorpromoting function [42]. High levels of M2 macrophages play a role in lymph node metastasis development. However, their distribution and clinical significance remain unclear. COX enzymes and PGE2 are expressed in penile cancer tissue [42]. They have immunosuppressive potency, and may aid in the formation of an immune hostile tumor micro-environment.

\section{Biomarkers}

Tumor cells can evade the immune system and its infiltrating cytotoxic CD8 T cells. The immune checkpoints, such as programmed cell death-1 (PD-1)/programmed cell death ligand-1 (PD-L1) and cytotoxic T lymphocyte antigen 4 (CTLA-4), may have a role in this process. PD-L1 expression is present in $40-60 \%$ of penile cancer patients [42]. Diffuse expression is associated with a worse outcome, whilst marginal PD-L1 expression is linked to a much better prognosis [45, 47]. Marginal PD-L1 expression may be initiated by stromal $\mathrm{T}$ cells, thus representing an active immune response, in contrast to diffuse PD-L1 expression which may be caused by genetic mutations [45]. Siglec- 15 was recently identified as another potential target and prognostic indicator in penile cancer [48].

A high neutrophil-to-lymphocyte ratio and C-reactive protein have been described as predictors for poor prognosis in penile cancer [39]. The production of the latter is induced by interleukin-6, a protein with immunosuppressive potency.

Human leukocyte antigens (HLAs) initiate the antitumor immune response by presenting tumor-associated peptides to T-cell receptors. Partial loss of HLA-A expression results in decreased overall survival in penile cancer patients [42].

\section{Imiquimod}

Stimulation of the host immune response against malignant cells has been employed in superficial penile cancer and precancerous lesions. Topical therapies can be used as a nonsurgical treatment modality for patients with PeIN [8]. The aim is to preserve the cosmesis and functionality of the penis. Imiquimod (IQ) and 5-fluorouracil (5-FU) are most commonly used as a topical ointment. IQ is an immune-modulating drug. It activates the adaptive immune through toll-like receptor 7 (TLR-7) which causes secretion of cytokines such as interferon alpha, interleukin-6 (IL-6) and tumor necrosis factor alpha [7]. Most data for the use of topical IQ in PeIN come from small case series. In a pooled review of 2017, Deen et al. included 48 patients treated with IQ [49]. Complete response was revealed in $62.5 \%$ of the patients, partial response in $8 \%$, and $29 \%$ demonstrated no response. The duration of treatment and frequency of application are variable in the studies, which makes it difficult to draw conclusions. Usually, the treatment frequency is 5 days a week for a period of 4 to 6 weeks. An important side effect of IQ is local toxicity, skin irritation can be outspoken. The results of imiquimod and 5-FU are comparable [8]. Insufficient response can signify underlying invasive disease. Hence, in case of non-responding to topical treatment, the lesion should be treated in a surgical manner. Re-challenge with topical products has to be avoided.

\section{Potential for immunotherapy}

Standard treatment modalities are limited to surgery, radiation and chemotherapy. Systemic therapeutic options for platinum-refractory, advanced penile cancer are limited. There is often a poor response to further systemic therapy. Metastatic cancer is associated with a dismal outcome with an overall survival of 6 to 12 months, and salvage treatments experience poor responses. Anemia and visceral metastases are important prognostic factors [50]. Immunotherapy continues to be explored in this setting; clinical trials are being employed to assess the role of immune checkpoint blockade in penile cancer. As mentioned before, MSI (> 20 mutations/ $\mathrm{Mb}$ ) was shown in $6 \%$ of the penile cancer specimens [40].

Preclinical data show that PD-L1 expression on infiltrating immune cells is present in $40-60 \%$ of the analyzed cases $[36,42]$. There is only anecdotal evidence on the activity of checkpoint inhibition in penile cancer. In a case series of 2019, a 79-year-old patient with metastatic penile cancer, which progressed after chemoradiation, was treated with PD-L1 inhibitor atezolizumab for approximately 2 years [51]. The treatment was discontinued after he developed immune-mediated toxicity. A restaging scan at 2 years showed near complete response.

Most trials including penile cancer patients are basket trials accruing rare solid or genitourinary malignancies [38]. The trials include combinations of PD-1/PD-L1 inhibition and CTLA-4 inhibitors. NCT02837042 included pembrolizumab for advanced penile cancer, NCT03333616/ NCT02834013 evaluate ipilimumab and nivolumab for advanced rare tumors including penile cancer.

Recently, Huang et al. from MD Anderson described a genetically engineered mouse (GEM) model of penile squamous cell carcinoma, manipulating through the pathways relevant to carcinogenesis mechanisms [52]. Mouse PeCa tumors promote an immunosuppressive micro-environment 
with dominantly myeloid-derived suppressor cells (MDSCs). In this model, preclinical trials show synergistic efficacy of immune checkpoint blockade with MDSC-diminishing drugs cabozantinib or celecoxib. Cisplatin resistance is induced by Pten deficiency and co-deletion of Smad4/ apc. The disadvantage to genetically engineered models is that the tumors are often monocloncal in nature and do not resemble the intra-tumoral heterogeneity seen in human penile cancer. To this end, recently developed xenograft models can be humanized to better identify biomarkers for immune checkpoint inhibitor responses [53].

\section{Conclusion}

The knowledge of molecular oncogenesis, with an important role of HPV, in penile cancer is expanding. This can lead to the identification of therapeutic targets which could significantly influence the prognosis of advanced penile cancer. Clinical trials in referral centers are being employed to pave the way for individualized treatment modalities.

Author contributions JV: data collection and manuscript writing. GV: data collection and manuscript writing. MA: manuscript writing and supervision.

\section{Compliance with ethical standards}

Conflict of interest No conflicts of interest to declare.

\section{References}

1. Hakenberg OW, Compérat EM, Minhas S, Necchi A, Protzel C, Watkin N (2015) EAU guidelines on penile cancer: 2014 update. Eur Urol 67(1):142-150

2. Douglawi A, Masterson TA (2017) Updates on the epidemiology and risk factors for penile cancer. Transl Androl Urol 6:785-790

3. de Martel C, Plummer M, Vignat J, Franceschi S (2017) Worldwide burden of cancer attributable to HPV by site, country and HPV type. Int J Cancer 141(4):664-670

4. Misra S, Chaturvedi A, Misra NC (2004) Penile carcinoma: a challenge for the developing World. Lancet Oncol 5(4):240-247

5. Thomas A, Vanthoor J, Vos G, Tsaur I, Albersen M (2020) Risk factors and molecular characterization of penile cancer: Impact on prognosis and potential targets for systemic therapy. Curr Opin Urol 300:202-207

6. Backes DM, Kurman RJ, Pimenta JM, Smith JS (2009) Systematic review of human papillomavirus prevalence in invasive penile cancer. Cancer Causes Control 20:449-457

7. Manjunath A, Brenton T, Wylie S, Corbishley CM, Watkin NA (2017) Topical Therapy for non-invasive penile cancer (Tis)updated results and toxicity. Transl Androl Urol 6:803-808

8. Raskin Y, Vanthoor J, Milenkovic U, Muneer A, Albersen M (2019) Organ-sparing surgical and nonsurgical modalities in primary penile cancer treatment. Curr Opin Urol 29:156-164
9. Moch H, Cubilla AL, Humphrey PA, Reuter VE, Ulbright TM (2016) The 2016 WHO classification of tumours of the urinary system and male genital organs-part A: Renal, penile, and testicular tumours. Eur Urol 70(1):93-105

10. Olesen TB, Sand FL, Rasmussen CL, Albieri V, Toft BG, Norrild B et al (2019) Prevalence of human papillomavirus DNA and p16 INK4a in penile cancer and penile intraepithelial neoplasia: a systematic review and meta-analysis. Lancet Oncol 20(1):145-158

11. Emmanuel A, Nettleton J, Watkin N, Berney DM (2019) The molecular pathogenesis of penile carcinoma-current developments and understanding. Virchows Arch 475:397-405

12. Eich ML, del Carmen Rodriguez Pena M, Schwartz L, Granada CP, Rais-Bahrami S, Giannico G et al (2020) Morphology, p16, HPV, and outcomes in squamous cell carcinoma of the penis: a multi-institutional study. Human Pathol 96:79-86

13. Klingelhutz AJ, Foster SA, McDougall JK (1996) Telomerase activation by the E6 gene product of human papillomavirus type 16. Nature 380(6569):79-82

14. De Paula AAP, Motta ED, Alencar RDC, Saddi VA, Da Silva RC, Caixeta GN et al (2012) The impact of cyclooxygenase-2 and vascular endothelial growth factor $\mathrm{C}$ immunoexpression on the prognosis of penile carcinoma. J Urol 187(1):134-140

15. Ferreux E, Lont AP, Horenblas S, Gallee MPW, Raaphorst FM, von Knebel DM et al (2003) Evidence for at least three alternative mechanisms targeting the $\mathrm{p} 16 \mathrm{INK} 4 \mathrm{~A} /$ cyclin $\mathrm{D} / \mathrm{Rb}$ pathway in penile carcinoma, one of which is mediated by high-risk human papillomavirus. Journal of Pathology 201(1):109-118

16. Poetsch M, Hemmerich M, Kakies C, Kleist B, Wolf E, Vom Dorp F et al (2011) Alterations in the tumor suppressor gene p16 INK4A are associated with aggressive behavior of penile carcinomas. Virchows Arch 458(2):221-229

17. Afonso LA, Carestiato FN, Ornellas AA, Ornellas P, Rocha WM, Cordeiro TI et al (2017) Human papillomavirus, Epstein-Barr virus, and methylation status of p16ink4a in penile cancer. J Med Virol 89(10):1837-1843

18. Kayes O, Ahmed HU, Arya M, Minhas S (2007) Molecular and genetic pathways in penile cancer. Lancet Oncol 8:420-429

19. Stankiewicz E, Prowse DM, Ng M, Cuzick J, Mesher D, Hiscock F et al (2011) Alternative HER/PTEN/Akt pathway activation in HPV positive and negative penile carcinomas. PLoS ONE 3:e17517

20. Ferrandiz-Pulido C, Masferrer E, Toll A, Hernandez-Losa J, Mojal S, Pujol RM et al (2013) MTOR signaling pathway in penile squamous cell carcinoma: pmTOR and peIF4E over expression correlate with aggressive tumor behavior. J Urol 190(6):2288-2295

21. Chaux A, Munari E, Cubilla AL, Hicks J, Lecksell K, Burnett AL et al (2014) Immunohistochemical expression of the mammalian target of rapamycin pathway in penile squamous cell carcinomas: a tissue microarray study of 112 cases. Histopathology 64(6):863-871

22. Hansen BT, Orumaa M, Lie AK, Brennhovd B, Nygård M (2018) Trends in incidence, mortality and survival of penile squamous cell carcinoma in Norway 1956-2015. Int J Cancer 142(8):1586-1593

23. Diorio GJ, Giuliano AR (2016) The role of human papilloma virus in penile carcinogenesis and preneoplastic lesions: a potential target for vaccination and treatment strategies. Urol Clin N Am 143:419-425

24. Shabbir M, Minhas S, Muneer A (2011) Diagnosis and management of premalignant penile lesions. Therapeut Adv Urol 3:151-158

25. Kidd LC, Chaing S, Chipollini J, Giuliano AR, Spiess PE, Sharma P (2017) Relationship between human papillomavirus and penile cancer-implications for prevention and treatment. Transl Androl Urol 6(5):791-802 
26. Djajadiningrat RS, Jordanova ES, Kroon BK, Van Werkhoven E, De Jong J, Pronk DTM et al (2015) Human papillomavirus prevalence in invasive penile cancer and association with clinical outcome. J Urol 193(2):526-531

27. Sand FL, Rasmussen CL, Frederiksen MH, Andersen KK, Kjaer SK (2018) Prognostic significance of HPV and p16 status in men diagnosed with penile cancer: a systematic review and meta-analysis. Cancer Epidemiol Biomark Prev 27:1123-1132

28. Ang KK, Harris J, Wheeler R, Weber R, Rosenthal DI, NguyenTân PF et al (2010) Human papillomavirus and survival of patients with oropharyngeal cancer. N Engl J Med 363(1):24-35

29. Rasmussen CL, Sand FL, Hoffmann Frederiksen M, Kaae Andersen K, Kjaer SK (2018) Does HPV status influence survival after vulvar cancer? Int J Cancer 142(6):1158-1165

30. Hölters S, Khalmurzaev O, Pryalukhin A, Loertzer P, Janssen M, Heinzelbecker J et al (2019) Challenging the prognostic impact of the new WHO and TNM classifications with special emphasis on HPV status in penile carcinoma. Virchows Arch 475(2):211-221

31. Schlenker B, Schneede P (2019) The role of human papilloma virus in penile cancer prevention and new therapeutic agents. Eur Urol Focus 5:42-45

32. Schabath MB, Villa LL, Lazcano-Ponce E, Salmerón J, Quiterio M, Giuliano AR (2012) Smoking and human papillomavirus (HPV) infection in the HPV in men (HIM) study. Cancer Epidemiol Biomark Prev 21(1):102-110

33. Schabath MB, Thompson ZJ, Egan KM, Torres BN, Nguyen A, Papenfuss MR et al (2015) Alcohol consumption and prevalence of human papillomavirus (HPV) infection among US men in the HPV in Men (HIM) study. Sex Transm Infect 91(1):61-67

34. Giuliano AR, Palefsky JM, Goldstone S, Moreira ED, Penny ME, Aranda C et al (2011) Efficacy of quadrivalent HPV vaccine against HPV infection and disease in males. N Engl $\mathrm{J}$ Med 364(5):401-411

35. Aydin AM, Cheriyan S, Spiess PE (2019) Treating advanced penile cancer: where do we stand in 2019? Curr Opin Supp Palliat Care 13:249-254

36. Aydin AM, Chahoud J, Adashek JJ, Azizi M, Magliocco A, Ross JS et al (2020) Understanding genomics and the immune environment of penile cancer to improve therapy. Nat Rev Urol 17:555

37. May M, Brookman-May SD, Ecke TH, Burger M (2018) Molecular characterization of penile cancer: literature review of new prognostic markers and potential therapeutic targets. Urologe 57:398-407

38. McGregor BA, Sonpavde GP (2020) Rare genitourinary malignancies: current status and future directions of immunotherapy. Eur Urol Focus 6:14-16

39. de Vries HM, Ottenhof SR, Horenblas S, van der Heijden MS, Jordanova ES (2019) Defining the tumor microenvironment of penile cancer by means of the cancer immunogram. Eur Urol Focus 5:718-721

40. Chalmers ZR, Connelly CF, Fabrizio D, Gay L, Ali SM, Ennis R et al (2017) Analysis of 100,000 human cancer genomes reveals the landscape of tumor mutational burden. Genome Med 9:34

41. Jacob JM, Ferry EK, Gay LM, Elvin JA, Vergilio JA, Ramkissoon $\mathrm{S}$ et al (2019) Comparative genomic profiling of refractory and metastatic penile and nonpenile cutaneous squamous cell carcinoma: implications for selection of systemic therapy. J Urol 201(3):541-548

42. Stoehr R, Wendler O, Giedl J, Gaisa NT, Richter G, Campean V et al (2019) No evidence of microsatellite instability and loss of mismatch-repair-protein expression in squamous cell carcinoma of the penis. Pathobiology 86(2-3):145-151

43. Feber A, Worth DC, Chakravarthy A, De Winter P, Shah K, Arya $\mathrm{M}$ et al (2016) CSN1 somatic mutations in penile squamous cell carcinoma. Can Res 76(16):4720-4727

44. Bonneville R, Krook MA, Kautto EA, Miya J, Wing MR, Chen $\mathrm{H}-\mathrm{Z}$ et al (2017) Landscape of microsatellite instability across 39 cancer types. JCO Precis Oncol 2017(1):1-15

45. Chu C, Yao K, Lu J, Zhang Y, Chen K, Lu J et al (2020) Immunophenotypes based on the tumor immune microenvironment allow for unsupervised penile cancer patient stratification. Cancers 12(7): 1796

46. Lohneis P, Boral S, Kaufmann AM, Lehmann A, Schewe C, Dietel $\mathrm{M}$ et al (2015) Human papilloma virus status of penile squamous cell carcinoma is associated with differences in tumour-infiltrating T lymphocytes. Virchows Arch 466(3):323-331

47. Ottenhof SR, Djajadiningrat RS, Thygesen HH, Jakobs PJ, Józwiak K, Heeren AM et al (2018) The prognostic value of immune factors in the tumor microenvironment of penile squamous cell carcinoma. Front Immunol. https://doi.org/10.3389/ fimmu.2018.01253

48. Wang J, Sun J, Liu LN, Flies DB, Nie X, Toki M et al (2019) Siglec-15 as an immune suppressor and potential target for normalization cancer immunotherapy. Nat Med 25(4):656-666

49. Deen K, Burdon-Jones D (2017) Imiquimod in the treatment of penile intraepithelial neoplasia: an update. Australas J Dermatol 58:86-92

50. Buonerba C, Di Lorenzo G, Pond G, Cartenì G, Scagliarini S, Rozzi A et al (2016) Prognostic and predictive factors in patients with advanced penile cancer receiving salvage (2nd or later line) systemic treatment: a retrospective, multi-center study. Front Pharmacol 7:487

51. Hui G, Ghafouri SN, Shen J, Liu S, Drakaki A (2019) Treating penile cancer in the immunotherapy and targeted therapy era. Case Rep Oncol Med 2019:1-4

52. Huang T, Cheng X, Chahoud J, Sarhan A, Tamboli P, Rao P et al (2020) Effective combinatorial immunotherapy for penile squamous cell carcinoma. Nature Commun 11(1):1-15

53. Thomas A, Vanthoor J, Himmelreich U, Cawthorne C, Deroose CM, Gsell W et al (2020) Establishment, characterization, and imaging of a first platinum-resistant penile cancer patientderived xenograft in nude mice: a eUROGEN project. Eur Urol 78:294-296

Publisher's Note Springer Nature remains neutral with regard to jurisdictional claims in published maps and institutional affiliations. 